\title{
Leucocyte adhesion deficiency presenting as a chronic ileocolitis
}

\author{
I D D'Agata, K Paradis, Z Chad, Y Bonny, E Seidman
}

\begin{abstract}
CD11/CD18 leucocyte glycoprotein deficiency is a rare, congenital adhesion molecule disorder which, in its severe form, is usually fatal. Leucocytes in affected subjects have abnormal migration and adherence, rendering patients susceptible to life threatening infections. The CD11/ CD18 integrins, and other adehsion molecules, are considered essential to the normal inflammatory response. It has been postulated that adhesion molecules may be responsible for mediating in part, the inflammatory changes observed in inflammatory bowel diseases and related disorders. This report describes the first case of CD11/CD18 deficiency characterised by a chronic ileocolitis. Bone marrow transplantation completely resolved the gastrointestinal symptoms, supporting a role for neutrophil dysfunction in the pathogenesis of the gut lesions. This case suggests that specific blockade of CD11/ CD18 integrins alone may not halt the chronic inflammatory response observed in immune mediated bowel disorders, and that abnormalities of leucocyte function must be included in the differential diagnosis of paediatric Crohn's disease.

(Gut 1996; 39: 605-608)
\end{abstract}

Keywords: leucocytes, adhesion molecules, ileocolitis.

I D D'Agata
K Paradis

E Seidman

and

Allergy/Immunology

Z Chad

Hôpital Ste-Justine, Montreal, Canada

Y Bonny

Bone Marrow

Transplant Unit, Hôpital Maisonneuvre-

Rosemont

Department of Paediatrics, University of Montreal, Faculty of Medicine, Montreal, Canada

Correspondence to:

Dr E G Seidman,

Division of Paediatric

Gastroenterology-Nutrition,

Hôpital Ste-Justine, 3175

Côte Ste-Catherine Road,

Montreal, Quebec H3T 1C5,

Canada.

Accepted for publication

16 May 1996

Considerable interest has recently been focused on adhesion molecules and their involvement in phagocyte-endothelial interactions and lymphocyte homing. ${ }^{12}$ Ligands for CD11/CD18 expressed on endothelial cells help mediate their interaction with polymorphonuclear cells (PMN) and monocytes. ${ }^{3}$ Expression of a number of leucocyte adhesion molecules such as LFA-1, Mac-1, ICAM-1 (CD54) are upregulated by pro-inflammatory cytokines and other mediators in inflammatory states. Quantitative abnormalities in adhesion molecules and the cytokines that induce their expression have been reported in patients with inflammatory bowel disease. ${ }^{4-7}$ Consequently, it has been postulated that specific blockade of adhesion molecules by monoclonal antibodies may attenuate the damage resulting from leucocyte activity at inflammatory sites, and thus be of therapeutic benefit to patients with inflammatory bowel disease. ${ }^{24}$
CD11/CD18 leucocyte glycoprotein deficiency is a rare, inherited disorder that presents in infancy under the guise of recurrent and often fatal bacterial infections. ${ }^{89}$ The leucocytes of affected patients are characterised by an absent or deficient expression of plasmalemma glcoproteins LFA-1 (CD11a/CD18), Mac-1 (CD11b/CD18), and p160,95 (CD11c/CD18), members of the leucocyte integrin family. These molecules are essential for many leucocyte adhesion related functions, including chemotaxis, aggregation, phagocytosis, and antibody directed cellular cytotoxicity. In the approximately 60 cases reported to date, the typical presentation was delayed separation of the umbilical cord, recurrent episodes of septicaemia, skin and wound infections, as well as severe gingival and periodontal disease. Gastrointestinal tract involvement has been reported in only a few cases, including appendicitis, peritonitis, ischaemic ileitis, and necrotising enterocolitis. ${ }^{10}{ }^{11}$ Inflammatory bowel diseaselike signs and symptoms have been described in several other congenital disorders of leucocyte function, ${ }^{12}$ such as chronic granulomatous disease. In this report, we provide the first description of a chronic ileocolitis in a patient with congenital CD11/CD18 deficiency that resolved after successful bone marrow transplantation.

\section{Case report}

A male born to non-consanguineous FrenchCanadian parents presented at three months of age with high fever, gastroenteritis, and a slowly healing lesion at the site of his first immunisation. Six admissions ensued over the following 15 months for episodes of fever of unknown origin, pustular and ulcerated lesions of the skin and lip, pneumonia, a perianal abscess requiring drainage, and balanopostitis resulting in phimosis. Each admission was characterised by high fevers (to $40^{\circ} \mathrm{C}$ ), leucocytosis (average of 28000 , up to 70000 cells/ $\mathrm{ml}$ ) with neutrophilia $(70-90 \%)$, and a slow response to multiple antibiotics. The immunological examination of this patient has been reported in detail previously (patient 1, reference 13).

Quantitative serum immunoglobulin values and complement assays were normal. Abnormalities included negative delayed hypersensitivity skin tests, and Rebuck skin windows showing no neutrophils or monocytes for up to 
24 hours on either of two occasions. A bone marrow biopsy was normal other than for myeloid hyperplasia. A biopsy of an ulcerated lesion of the lower lip revealed stromal infiltration by mononuclear cells, with few neutrophils. An unanticipated adverse reaction occurred after the biopsy procedure, with massive swelling of the lip, such that even liquid intake became difficult. Complete deficiency of all three molecules of the CD11/ CD18 complex was confirmed using both FACS and immunoprecipitation techniques, with monoclonal antibodies.

Between 1.5 and 5 years of age, he required treatment for recurrent otitis media, ulcerative stomatitis, and periodontal disease. Poor weight gain due to inadequate food intake was attributed to abdominal and periodontal pain. At 5.5 years of age, he was referred to our Paediatric Gastroenterology service because of failure to thrive and recurrence of a perianal abscess. Family history was negative for inflammatory bowel disease. His weight had fallen below the third percentile for age. Barium studies (upper gastrointestinal, small bowel follow through, and barium enema) revealed involvement of the terminal ileum, caecum, and ascending colon, resembling Crohn's ileocolitis (Figure). At sigmoidoscopy, a hyperaemic mucosa was noted, without ulcerations. Biopsy specimens were not obtained because of the previous complications of impaired healing after his lip biopsy. Treatment with mesalamine (Asacol, $30 \mathrm{mg} / \mathrm{kg} /$ day) was started, along with metronidazole, resulting in a decrease in both stool frequency and abdominal cramping. Inadequate weight gain persisted despite high energy oral supplements, and he was discharged home in improved clinical condition receiving mesalamine and nocturnal nasogastric (Vivonex) feedings.

Six months later, he was readmitted to hospital for fever, severe perianal disease, and a relapse of his diarrhoea. Blood cultures grew $S$ epidermidis and an Enterobacter species, and a urine culture was positive for $S$ epidermidis. Physical examination showed splenomegaly, as well as another perianal abscess and fistula. His anaemia $(7.2 \mathrm{~g} / \mathrm{dl})$ required transfusions. Colonoscopy now showed multiple aphthous ulcers dispersed throughout the colon. The

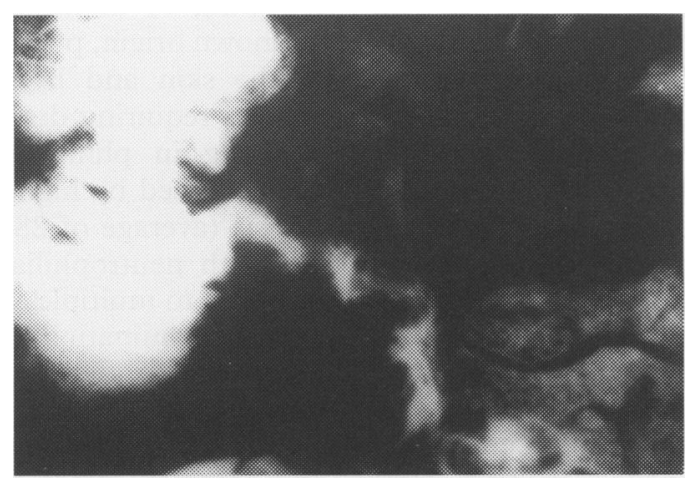

Ileocolitis in a patient with CD11/CD18 leucocyte

glycoprotein deficiency. The terminal ileum and caecum are characterised by oedematous mucosal folds and spasticity, as commonly seen in Crohn's disease. ileal mucosa was also ulcerated, with copious fibrinous exudates and pseudopolyps. No pseudomembranes were seen, and stools were pathogen and $C$ difficile toxin negative. An oesophagogastroduodenoscopy showed hyperaemia of the antrum and aphthous ulcers in the antrum and duodenal bulb. During this admission, he developed inspiratory stridor and hypoxaemia, requiring intubation and ventilatory assistance. Pseudomonas aeruginosa tracheitis was diagnosed, and a tracheotomy was performed because of prolonged intubation and assisted ventilation requirements. The tracheotomy wound healed slowly, and antibiotics were unsuccessful in eradicating the pseudomonas infection until granulocyte transfusions were administered.

Because of his progressively deteriorating clinical condition, a bone marrow transplantation was performed at 7 years of age. Immunosuppressive therapy given in the peritransplant period included BCMU $\left(300 \mathrm{mg} / \mathrm{m}^{2}\right.$ intravenous once), cyclophosphamide $(35 \mathrm{mg} /$ $\mathrm{kg} / \mathrm{d}$ intravenous for four days), VP-16 (100 $\mathrm{mg} / \mathrm{m}^{2}$ every 12 hours intravenous for four days), and ARA-C (100 mg/m $\mathrm{m}^{2}$ every 12 hours for four days). Cyclosporine $(5 \mathrm{mg} / \mathrm{kg}$ ) and prednisone $(1 \mathrm{mg} / \mathrm{kg}$ ) were administered orally for the first six months, and then tapered gradually over the following six month period. Only one minor episode of graft versus host disease was noted two weeks after the allogenic bone marrow transplant. The diarrhoea and all other gastrointestinal symptoms resolved completely within a few weeks after bone marrow transplant. A repeat colonoscopy confirmed a normal mucosa, with complete healing of all ulcers. He has remained symptom free for over a three year follow up, without immunosuppression for the last two years.

\section{Discussion}

Unlike the previously described cases of colitis in CD11/CD18 deficiency attributable to ischaemic causes with an acute onset, ${ }^{11}$ our patient's enterocolitis presented insidiously over the course of three years. Signs and symptoms suggestive of a Crohn's disease-like disorder included diarrhoea, abdominal pain, and recurrent perianal disease. Extraintestinal manifestations suggestive of an inflammatory bowel disease-like disorder were intermittent fevers, aphtous stomatitis, and growth failure. Radiological and endoscopic findings also resembled Crohn's ileocolitis. The diarrhoea improved with mesalamine and metronidazole treatment, as might be anticipated in inflammatory bowel disease. However, this patient clearly did not have Crohn's disease, as the current definition of inflammatory bowel disease implies an idiopathic state of chronic intestinal inflammation, excluding all known secondary causes, including an immune deficiency state. ${ }^{12}$

Congenital CD11/CD18 deficiency was initially described in 1974 by Boxer and colleagues, ${ }^{14}$ and in 1980 , Crowley et al ${ }^{15}$ first proposed its molecular basis. These molecules consist of a distinct $\alpha$ subunit (CD11a or 
CD11b or CD11c), and a common $\beta$ subunit (CD18). Each member of the CD11/CD18 family mediates specific, as well as overlapping leucocyte adhesion related functions. ${ }^{1-3} \mathrm{Ab}-$ normal expression of the gene for the $\beta$ subunit results in an inability of normal surface membrane expression of these three heterodimers. Two phenotypic variants of the disorder exist, based on the degree to which CD11/CD18 expression is impaired. ${ }^{11}$

The case presented in this report may be regarded as an accident of nature that allows us to examine the consequences of human CD11/CD18 'gene knock-out' from the gastrointestinal point of view. Chronic diarrhoea, often accompanied by malabsorption and failure to thrive, is characteristic of several primary immunodeficiency syndromes. ${ }^{16}$ Other congential disorders of neutrophil function may also present with an entercolitis resembling Crohn's disease, as in patients with chronic granulomatous disease, ${ }^{17}$ glycogen storage disease type $1 b,{ }^{18}$ and the HermanskyPudlak syndrome. ${ }^{19}$ Although neutrophil function is normal in the latter syndrome, the intestinal lesions may be secondary to a defect in macrophage function due to a toxic accumulation of a ceroid-like pigment. ${ }^{20}$

It is intriguing that diverse abnormalities of leucocyte function may present with the same Crohn disease-like enterocolitic picture. ${ }^{16}$ It is generally held that the bacterial flora plays a part in the pathogenesis of Crohn's disease. ${ }^{21}$ Leucocytes help maintain normal gastrointestinal mucosal homeostasis, in part by impeding the translocation of intraluminal bacteria, particularly in the context of abnormal intestinal permeability in the setting of intestinal inflammation. Evidence linking the enteric flora to the pathogenesis of inflammatory bowel disease has been provided by experimental models such as interleukin 10 and interleukin 2 knockout mice, and HLAB27 transgenic rats, in whom the severe intestinal inflammation is not seen under gnotobiotic conditions. ${ }^{22}$ It thus seems that subjects incapable of mounting an appropriate mucosal response to endogenous bacteria, in the presence of an inherited leucocyte dysfunction, are prone to develop chronic intestinal inflammation. ${ }^{23}$ That the immune defect at the heart of CD11/CD18 deficiency was responsible for our patient's gastrointestinal symptomatology seems probable, as all symptoms resolved permanently once bone marrow transplant was performed. This improvement may theoretically have resulted from the immunosuppressive treatment the patient received before, and after, his bone marrow transplant. However, he has remained completely free of gastrointestinal symptoms for three years since bone marrow transplant, two of which were without immunosuppressive use.

There is considerable interest in the potential therapeutic implications that various adhesion molecules may have in the pathogenesis of clinical conditions characterised by a changed immune function, including inflammatory bowel disease. Adhesion mole- cule expression on leucocyte and endothelial cells is inducible during the course of inflammation in response to cytokines, lipopolysaccharide, and oxygen free radicals. ${ }^{24}{ }^{25} \mathrm{Up}$ regulation of adhesion molecules facilitates traffic of leucocytes to sites of inflammation, thereby determining in part the extent and nature of the inflammatory response. The upregulation of leucocyte adhesion molecule expression by tumour necrosis factor $\alpha$ can be inhibited by sulphasalazine, suggesting that this may be a mechanism by which such treatment prevents leucocyte recruitment into parenchymal tissue in inflammatory bowel disease. ${ }^{26}$ It has also been proposed that antibodies to integrins may help diminish tissue damage at inflammatory sites by inhibiting adhesion and transmigration of leucocytes across the endothelium. ${ }^{27}$ Anti-CD11b/ CD18 monoclonal antibodies have been shown to attenuate the consequences of experimentally induced ischaemic reperfusion injury. ${ }^{28} 29$ However, other CD11/CD18 independent pathways of inflammation have been shown to exist. ${ }^{30}$ Furthermore, in another leucocyte disorder in which integrin expression is normal but the selectin system is crippled, gastrointestinal symptoms are not prominent. ${ }^{31}$ Obviously, anti-CD11/CD18 monoclonal antibodies in our patient would have been of no benefit whatsoever. This does not guarantee that anti-CD11/CD18 monoclonal antibody therapy is devoid of benefit in Crohn's disease, as the pathological mechanisms involved in leucocyte adhesion deficiency associated ileocolitis may differ from those operating in Crohn's disease.

In this report, we have described the development of an inflammatory bowel diseaselike illness in a child with congenital CD11/ CD18 deficiency. This disorder thus represents yet another example of a primary immunodeficiency syndrome involving neutrophils, which may present with a non-specific enterocolitis. Disorders of neutrophil function should be included in the differential diagnosis of paediatric Crohn's disease, ${ }^{12}{ }^{16}$ particularly in the presence of severe stomatitis and periodontal disease. This case also suggests that, to control inflammation in inflammatory bowel disease, targeting one adhesion molecule, such as LFA-1 of the CD11/CD18 complex, is probably not sufficient.

Supported by Chercheur Boursier Clinicien Research Award from the Fonds de Recherche en Santé du Quebec (KP, ES) and the Astra Canadian Association of Gastroenterology Research Initiative Award (ID, ES).

\footnotetext{
Springer TA. Adhesion molecules of the immune system Nature 1990; 346: 425-34.

2 Albelda SM, Smith CW, Ward PA. Adhesion molecules and inflammation injury. FASEB $\mathcal{F}$ 1994; 8: 504-12.

3 Stoolman LM. Adhesion molecules controlling lymphocyte migration. Cell 1989; 56: 907-10.

4 Salmi M, Gransfors K, MacDermott R, Jalkanen S Aberrant binding of lamina propria lymphocytes to vascular endothelium in inflammatory bowel diseases. Gastroenterology 1994; 106: 596-605.

5 Koizumi OH, King N, Lobb R, Benjamin C, Podolsky DK Expression of vascular adhesion molecules in inflammatory bowel disease. Gastroenterology 1992; 103: 840-7.

6 Nielsen OH, Langholz E, Hendel J, Brynskov J. Circulating soluble intercellular adhesion molecule-1 (sICAM-1) in active inflammatory bowel disease. Dig Dis Sci 1994; 39:
} 1918-23. 
7 Schuerman GM, Aber-Bishop AE, Facer P, Lee JC, Rampton DS, Dore CJ, et al. Altered expression of cell ampion molecules in uninvolved gut in inflammatory adhesion molecules in uninvolved gut in inflam

8 Todd RF, Freyer DR. The CD11/CD18 leukocyte glycoprotein deficiency. Hemat Oncol Clin North Am 1988; 2: 13-31.

9 Arnaout MA. Structure and function of the leukocyte adhesion molecules CD11/CD18. Blood 1990; 75: 1037-49.

10 Hawkins HK, Heffelfinger SC, Anderson DC. Leukocyte adhesion deficiency: clinical and postmortem observations. Pediatr Pathol 1992; 12: 119-30.

11 Anderson DC, Schmalsteig FC, Finegold MJ, Hughes BJ, Rothlein R, Miller LJ, et al. The severe and moderate phenotypes of heritable Mac-1, LFA-1 deficiency: their phenotypes of heritable and relation to leukocyte dysquantitative definition and relation to leukocyte dysfunction

12 Seidman EG. Inflammatory bowel diseases. In: Roy CC, Silverman A, Alagille D, eds. Pediatric clinical gastroenterology. 4th ed. St Louis: Mosby Press, 1995: 417-93.

13 Wright SD, Detmerg PA, Aida Y, Adamowski R Anderson DC, Chad Z, et al. CD18-deficient cells respond to lipopolysaccharide in vitro. $\mathcal{F}$ Immunol 1990; 144: 2566-71.

14 Boxer LA, Hedley-Whyte ET, Stossel TP. Neutrophil actin dysfunction and abnormal neutrophil behaviour. $N$ Engl fMed 1974; 291: 1093-9.

15 Crowley CA, Curnulte JT, Rosin RE, André-Schwartz J, Gallin JI, Klempner M, et al. An inherited abnormality of neutrophil adhesion: its genetic transmission and its 302: $1163-8$

16 Gurbindo C, Seidman EG. Gastrointestinal manifestations of primary immunodeficiency diseases. In: Walker WA Durie PR, Hamilton JR, Walker-Smith JA, Watkins JB, eds. Pediatric gastrointestinal disease: pathophysiology, diagnosis, management. 2nd ed. Philadelphia: B C Decker, 1996: 585-605.

17 Werlin SL, Chusid MJ, Oechler HW. Colitis in chronic granulomatous disease. Gastroenterology 1982; 82: 328-31.

18 Couper R, Kapelushnik DL, Griffiths AM. Neutrophil dysfunction in glycogen storage disease Ib: association dysfunction in glycogen storage disease Ib: association 549-54.

19 Schinella RA, Alba Greco M, Cobert BL, Denmark LW, Cox RP. Hermansky-Pudlak syndrome with granulomatous colitis. Ann Intern Med 1980; 92: 20-3.
20 Shanahan F, Randolph L, King R, Oseas R, Brogan M, Witkop $\mathrm{C}$, et al. Hermansky-Pudlak syndrome: an immunologic assessment of 15 cases. Am $\mathcal{f}$ Med $1988 ; 85$. 823-8.

21 Sartor RB. Microbial factors in the pathogenesis of Crohn's disease, ulcerative colitis, and experimental intestinal inflammation. In: Kirsner JB, Shorter RJ, eds Inflammatory bowel disease. 4th ed. Baltimore, Williams and Wilkins, 1995: 96-124.

22 Sartor RB. Current concepts in the etiology and the pathogenesis of ulcerative colitis and Crohn's disease. Gastroenterol Clin North Am 1995; 24: 475-505.

23 Strober W, Erhardt RO. Chronic intestinal inflammation: an unexpected outcome in cytokine or T-cell receptor mutant mice. Cell 1993; 75: 203-5.

24 Kishimoto TK, Anderson DC. The role of integrins in inflammation. In: Gallin JI, Goldstein IM, Snyderman R, eds. Inflammation: basic principles and clinical corre

25 Yan H, Juhasz I, Plewsky J, Murphy GF, Herlyn M Albelda SM. Human severe combined immunodeficien mouse chimeras. An experimental in vivo model system to study the regulation of human endothelial cell leukocyte adhesion molecules. $\mathcal{F}$ Clin Invest 1993; 91: 986-96.

26 Greenfield SM, Hamblin AS, Shakoor ZS, Teare JP, Punchard NA, Thompson RPH. Inhibition of leukocyte adhesion molecule upregulation by tumor necrosis factor $\alpha$ : a novel mechanism of action of sulphasalazine. Gut 1993; 34: 252-6.

27 Dana N, Arnaout MA. Leukocyte adhesion molecular (CD11/CD18) deficiency. In: Kazatchine M, ed. Baillier's clinical immunology and allergy (vol 2). Philadelphia: clinical immunology and

28 Vedder NB, Winn RK, Rice CL, Chi EY, Arfors KE Harlan JM. A monoclonal antibody to the adherence promoting leukocyte glycoprotein, CD18, reduced organ injury and improves survival from hemorrhagic shock in rabbits. F Clin Invest 1988; 81: 939-44.

29 Haskard D, Cavender D, Beatty P, Springer T, Ziff M Lymphocyte adhesion to endothelial cells: mechanisms demonstrated by anti-LFA 1 monoclonal antibodies. $\mathcal{F}$ Immunol 1986; 137: 2901-6.

30 Doerschuk CM, Winn RK, Coxson HO, Harlam JM CD18dependent and independent mechanisms of neutrophil dependent and independent mechanisms of neutrophil migration in the pulmonary and systemic mic
of rabbits. F Immunol 1990; 144: 2327-33.

31 Etzioni A, Frydman M, Pollack S, Avidor I, Phillips ML, Paulson JC, et al. Brief report: recurrent severe infections caused by a novel leukocyte adhesion deficiency. $N$ Engl f Med 1993; 327: 1789-92. 